\title{
RELATIONSHIP BETWEEN ASCARIS LUMBRICOIDES INFECTION AND BRONCHIAL ASTHMA IN JUHAYNA CITY, SOHAG, UPPER EGYPT
}

\section{By}

\author{
AMAL M. AHMED ${ }^{1 *}$ and NASR EL DEEN M. M. ALI ${ }^{2}$, \\ Departments of Medical Parasitology, Faculties of Medicine, Sohag University ${ }^{1}$ \\ Sohag and Al-Azhar University ${ }^{2}$, Assiut Branch, Egypt \\ ( ${ }^{\star}$ Correspondence: moustafad658@yahoo.com)

\section{ABSTRACT}

Bronchial asthma is a chronic inflammatory disorder of the airways characterized by airway hyper responsiveness and reversible airflow obstruction that fluctuates over time. Parasitic infection is one of the environmental factors associated with a small increase in bronchial asthmatic risk. In the present study, 200 stool samples were collected from patients suffering from bronchial asthma and 200 stool samples from non-asthmatic healthy volunteers as control group. The samples were preserved in $10 \%$ formalin and examined macroscopically, microscopically by direct smear \& formol ether sedimentation techniques. It was found that $12 / 200$ cases $(6 \%)$ were infected with Ascaris lumbricoides in asthmatic group and 2/200 cases (1\%) in control $(\mathrm{P}=0.007)$ illustrating significant difference between cases and control group indicating a role of Ascaris lumbricoides as a risk factor for bronchial asthma.

Key words: Bronchial asthma, Ascaris lumbricoides, Juhayna, Sohag

\section{Introduction}

Bronchial asthma is a chronic inflammatory disorder of the airways characterized by airway hyper responsiveness and reversible airflow obstruction that fluctuates over time (Anderson, 2008). Asthma is caused by a combination of complex and incompletely unknown environmental and genetic interactions (Martinez, 2007). Many environmental factors were associated with development of asthma and exacerbation including allergens, air pollution and other environmental chemicals (Kelly and Fussell, 2011). Asthma is associated with exposure to indoor allergens which commonly include dust mites, cockroaches, animal dander (fragments of fur or feathers), and mold (Arshad, 2010). Infection with parasites is one of environmental factors which may be associated with a small increase in asthma risk (Leonardi-Bee et al, 2006) and Ascaris lumbricoides was blamed to be a risk facttor in bronchial asthma (Alcantara-Navis et al, 2010; Bragagnoli, and Silva, 2014).

The present work aimed to study the relationship between the ascariasis infection and development of bronchial asthma in Juhayna City, Sohag Governorate, Egypt.

\section{Materials and Methods}

In the present study, 200 stool samples were collected from patients suffering from bronchial asthma and 200 stool samples from non-asthmatic healthy cross matched volunteers as control group from outpatient clinic of Juhayna Hospital, Sohag, from March to October 2016. Written consent was taken from all participated patients, which was approval by the ethics Committee of Scientific Research of the Faculty of Medicine, Sohag University.

Fresh stool samples were collected in clean dry plastic labeled containers, examined macroscopically for consistency, presence blood or mucus and parasites (round worms or tapeworm proglottids). Stool samples were examined by direct smear and formol ether sedimentation methods for parasites and cultured to avoid strogyloidiasis infected ones and Modified ZN to avoid Cryptosporidium parvum and Microsporidium species (Garcia et al, 2014).

Statistical analysis: Data were tabulated and analyzed using the SPSS version, 16. For the quantitative data, mean and standard deviation were calculated. The Chi-square test $\left(\chi^{2}\right)$ was used to compare frequency. If $P$ value $<0.05$ it was considered significant 
and $\mathrm{P}<0.001$ was considered highly significant.

In this study, twelve out of 200 asthmatic cases (6\%) were infected with Ascaris lumbricoides while only two out of 200 cases
$(1 \%)$ in control group $(\mathrm{P}=0.007)$ with significant difference between patients and controls (Tab. 1).

\section{Results}

The results were given in tables $(1 \& 2)$.

\begin{tabular}{|c|c|c|c|}
\hline Parameter & Asthmatic group $(\mathrm{N}=200)$ & Control group $(\mathrm{N}=200)$ & P-value \\
\hline Positive & $12(6 \%)$ & $2(1 \%)$ & $0.007 *$ \\
Negative & $188(94 \%)$ & $198(99 \%)$ & \\
\hline \multicolumn{2}{|c|}{ P-value high significant } \\
Table 2: Univariate logistic regression analysis. \\
\hline \\
Characteristics & OR (CI $95 \%)$ & P - value \\
\hline
\end{tabular}

A. lumbricoides infection was associated with significantly increased odds of bronchial asthma $(\mathrm{OR}=6.3)$

\section{Discussion}

The helminthes particularly nematodes as a cause of pulmonary disease and asthmatic bronchitis in Egyptian children were reported (Habib et al. (2017).

In this connection, Weiss (2000) reviewed the role of parasites in inducing asthma/allergy and concluded that the relation between infection with helminthic parasites and the development of asthma and allergy was a complicated medical problem and Pereira et al. (2007) reported that most bronchial asthmatic cases were of the non-atopic phenotype suggesting that some helminths might exert an attenuating effect on the expression of the disease atopic portion. Later on, Kunst et al. (2011) reported that parasitosis must be in the differential diagnosis of lung diseases and Santiago et al. (2015) confirmed that helminthic infection alters $\operatorname{IgE}$ responses to the allergens related to the parasite protein. Palmer et al. (2002) and Scott (2008) suggested that a complex relationship between ascariasis and susceptibility to the childhood asthma.

The present results showed that the prevalence of A. lumbricoides in asthmatic group $(6 \%)$ and in control group $(1 \%)$ which was highly significant $(\mathrm{P}=0.007)$ showing that A. lumbricoides infection is one of the risk factor for bronchial asthma (odds ratio = 6.3). The present results agreed with those of Leonardi-Bee et al. (2006) who found that A. lumbricoides was associated with significant increase in the bronchial asthma. Palm- er et al. (2002) in China reported the increased risk of childhood asthma in association with ascariasis infection. Hunninghake et al. (2007) found that ascariasis contributed to an increased risk of asthma either by causing inflammation in airways during larval migration or by increased atopic allergy. Hawlader et al. (2014) in Bangladesh found that ascariasis was risk factor for asthma and atopic allergy in rural area.

No doubt, theTh2 immune response reacts to helminthiasis infection and the allergic diseases such as asthma, rhinitis and eczema (Kay, 2001). Helminthiasis stimulate preferentially inflammatory Th2 type immune responses in patients (Cooper et al, 2009) by production of high levels of serum specific IgE and allergic reactivity toward parasite soluble antigens, which led to the development of bronchial hyper reactivity and asthma particularly in atopic individuals (Hagel et al, 2007). There were close similarities between the allergic inflammatory responses stimulated by environmental allergens and immune responses elicited by parasite antigens (Hopkin, 2009). Caraballo et al. (2015) confirmed that ascariasis modified the natural history of asthma, increasing Th2 responses and IgE synthesis to cross-react and species-specific dust mites' allergens as a risk factor for asthma and its severity. Buendia et al. (2015) stated that IgE sensitivity to ascariaisis and cross-reactive tropomyosins were frequent associated with clinical indicators of asthma severity with significant 
relationship between sensitization to nematode specific marker Asc s1 and ER attendance.

Shalaby and Shalaby (2016) in Mansoura reported that $A$. lumbricoides infection was associated with significantly higher levels of IL-4, IL-5 cytokines.

The present study disagreed with the study done by Dagoye et al. (2003) who revealed that Ascaris infection was associated with significant decreased wheezing risk. They provided evidence that systematicphase parasites could protect against the asthma manifestations afforded even in a very young children; involving probably enhanced down regulatory molecules such as IL $10 \&$ transforming growth factor (TGF $\beta$ ) and/or suppress ion of pro-inflamatory interlukins IL 4, IL 5 and the eotaxin.

Zakzouk et al. (2018) found that ascariasis infection exerted both the risk and protective effects on the asthma symptoms associated with the infection severity and genetic factors manifested by infection degree and asthma symptoms.

\section{Conclusion}

The present study found significant relationship between the Ascaris lumbricoides and bronchial asthma.

No doubt, the asthmatic bronchitis development and ascariasis could be affected by other factors as age, repeated infection, worm burden, genetic factors and low sanitary conditions in rural areas which are ongoing studies.

\section{Acknowledgements}

Many thanks are due to the Staff Members of Juhayna Hospital, Sohag for allowing and facilitating this applied work.

Thanks are also due to Dr. Refaat M. A. Khalifa, Professor of Medical Parasitology, Faculty of Medicine, Assiut University, for kindly revising the paper.

\section{References}

Anderson, GP, 2008: Endotyping asthma: new insights into key pathogenic mechanisms in a complex, heterogeneous disease. Lancet, 372, 9643:1107-19.
Alcantara-Neves, NM, Badaro, SG, dos Santos, MC, et al, 2010: The presence of serum anti- Ascaris lumbricoides IgE antibodies and of Trichuris trichiura infection as risk factors for wheezing and/or atopy in preschool-aged Brazilian children. Respir. Res. 23, 11:114-20

Arshad, SH, 2010: Does exposure to indoor allergens contribute to the development of asthma and allergy? Curr. Allergy Asthma Rep. 10, 1:49-55.

Bragagnoli, G, Siva, MT, 2014: Ascaris lumbricoides infection and parasite load are associated with asthma in children. J. Infect. Dev. Ctries 8, 7:891-7.

Buendia E, Zakzouk J, Mercado D et al. 2015: The IgE response to Ascaris molecular components is associated with clinical indicators of asthma severity, WAO J. 8, 8:1-11

Caraballo L, Acevedo N, Buendia E. 2015: Human ascariasis increases the allergic responses and allergic symptoms. Curr, Trop, Med. Rep. 2:224-32.

Cooper. P, Rodrigues, L, Cruz, A, Barreto M, 2009: Asthma in Latin America: A public health challenge and research opportunity. Allergy 64, 1:5-17.

Dagoye, D, Bekele, Z, Woldemichael, K, et al, 2003: Wheezing, allergy, and parasite infection in children in urban and rural Ethiopia. Am. J. Respir. Crit. Care Med. 167, 10:1369-73.

Garcia, LS, Arrowood, M, Kokoskin, E, Paltridge, G, Pillai, D, et al, 2014: Laboratory diagnosis of parasites from the gastrointestinal tract. Clin. Microbiol. Rev. DOI: 10.1128/CMR.

Habib, Y, Shaheen, M, Zidan, M, et al, 2017: Relation between bronchial asthma and parasitic (Nematodes) infection in Egyptian children. Pediatrics and Primary care Physicians (PPCP), 1, $1: 10-2$

Hagel, I, Cabrera, M, Hurtado, M et al, 2007: Infection by Ascaris lumbricoides and bronchial hyper-reactivity: an outstanding association in Venezuelan school children from endemic areas. Acta Trop, 103:231-41

Hopkin, J, 2009: Immune and genetic aspects of asthma, allergy and parasitic worm infections: evolutionary links. Parasit. Immunol. 31, 5:26773.

Hunninghake, GM, Soto-Quiros, ME, Avila, L, et al, 2007: Sensitization to Ascaris lumbricoides and severity of childhood asthma in Costa Rica. J. Aller. Clin. Immunol. 119: 654-61. 
Kay, A, 2001: Allergy and allergic diseases: First of two parts. N. Engl. J. Med. 344, 1:30-7.

Kelly, FJ, Fussell, JC, 2011: Air pollution and airway disease. Clin. Exp. Aller. 41, 8: 1059-71

Kunst, H, Mack, D, Kon, OM, et al, 2011: The parasitic infections of the lung: a guide for the respiratory physician. Thorax, 66:528- 36

Leonardi-Bee, J, Pritchard, D, Britton, J, 2006: Asthma and current intestinal parasite infection, Am. J. Respir. Crit. Care Med. 174, 5: 514-23.

Martinez, FD, 2007: Genes, environments, development and asthma: A reappraisal. Eur. Respir. J. 29, 1:179-84.

Palmer, LJ, Celedon, JC, Weiss, ST, et al, 2002: Ascaris lumbricoides infection is associated with increased risk of childhood asthma and atopy in rural China. Am. J. Respir. Crit. Care Med. 165:1489-93
Santiago, H, Flavia, L, Bennuru, R, et al, 2015: Helminth infection alters $\operatorname{IgE}$ responses to allergens structurally related to parasite protein. J. Immunol. 194:93-100

Scott, ME, 2008: Ascaris lumbricoides: A review of its epidemiology and relationship to other infections. Ann. Nestle (Engl.), 66:7-22

Shalaby, NH, Shalaby, NM, 2016: Effect of Ascaris lumbricoides infection on T-helper cell type 2 in rural Egyptian children. Therap. Clin. Risk Manag. 9, 12:379- 85

Weiss, ST, 2000: Parasites and asthma/ allergy. What is the relationship? J. Aller. Clin. Immunol. 105:205-10

Zakzouk, J, Casadiego, S, Mercado, A, et al, 2018: Ascaris lumbricoides infection induces reduction and increase of asthma symptoms in a rural community. Acta Trop. 197:1-4. 